\title{
RESTAURAÇÃO FLORESTAL NA MATA ATLÂNTICA: PASSIVA, NUCLEAÇÃO E PLANTIO DE ALTA DIVERSIDADE
}

\section{FOREST RESTORATION IN THE ATLANTIC FOREST: PASSIVE RESTORATION, NUCLEATION AND HIGH DIVERSITY PLANTATION}

\author{
Bruna Elisa Trentin ${ }^{1}$ Daniela Aparecida Estevan ${ }^{2}$ Elson Felipe Sandoli Rossetto ${ }^{3}$ \\ Mauricio Romero Gorenstein ${ }^{4}$ Gilmar Poser Brizola ${ }^{1}$ Fernando Campanhã Bechara ${ }^{4}$
}

\section{RESUMO}

Devido à crescente degradação ambiental, entender o funcionamento das técnicas de reflorestamento e outras técnicas de restauração tornou-se substancial para embasar a recomposição destes ecossistemas degradados. O objetivo deste trabalho foi monitorar a vegetação de uma área em restauração através de três diferentes técnicas. A área possui 7,2 ha em floresta subtropical em Dois Vizinhos, estado do Paraná, Brasil e encontra-se a aproximadamente 20 metros de um fragmento de floresta secundária. O experimento foi delineado através de 12 parcelas com três tratamentos $\left(T_{1-3}\right)$, distribuídas em quatro blocos casualizados. $\mathrm{T}_{1}$ : regeneração natural através da restauração passiva. $\mathrm{T}_{2}$ : conjunto de técnicas de nucleação. $\mathrm{T}_{3}$ : plantio de mudas através de linhas de recobrimento e diversidade ( 3 x $2 \mathrm{~m}$ ). Para fins de amostragem, cada parcela foi dividida em 24 subparcelas. Com a finalidade de avaliar a presença de plantas, após dois anos e oito meses da implantação dos tratamentos foi realizado um levantamento em três subparcelas aleatórias para cada parcela, em que foram identificados e quantificados todos os indivíduos maiores que $50 \mathrm{~cm}$ de altura. A riqueza de espécies nativas foi acima de $87 \%$ em todos os tratamentos, enquanto a presença de espécies herbáceas foi maior na restauração passiva e a de lenhosas no plantio. As síndromes de dispersão mais comuns foram anemocoria e zoocoria. Através da comparação entre os indivíduos plantados e regenerantes dos três tratamentos, a técnica de plantio apresentou a maior diversidade de Shannon $(3,017)$ com 148 indivíduos e 53 espécies, entretanto, não houve diferença significativa. Essa maior diversidade está associada ao grande número de espécies plantadas, mas ainda não há a facilitação da regeneração de espécies em seu sub-bosque, nesta idade. Analisando-se apenas os indivíduos regenerantes, o tratamento que obteve maior diversidade de Shannon foi a nucleação $(2,078)$ com um total de 908 indivíduos pertencentes a 40 espécies, contudo, esta não foi estatisticamente diferente da restauração passiva (Shannon $=1,965$ ) que apresentou 2.018 indivíduos pertencentes a 40 espécies. Os tratamentos mais similares floristicamente, foram a restauração passiva e a nucleação. A nucleação facilitou a regeneração natural de espécies (17 espécies plantadas e 40 regenerantes) enquanto a restauração passiva evidenciou alta resiliência, desta forma, a utilização da restauração passiva pode ser considerada uma eficiente alternativa, uma vez que seus resultados não se diferiram estatisticamente da nucleação, e a principal vantagem desta técnica é o custo reduzido.

Palavras-chave: técnicas nucleadoras; linhas de recobrimento e diversidade; restauração ecológica.

1 Engenharia Florestal, Universidade Tecnológica Federal do Paraná, Campus Universitário, CEP 85660-000, Dois Vizinhos (PR), Brasil. brunaelisatrentin@gmail.com / gil_mar12@hotmail.com

2 Bióloga, Dra., Professora da Coordenação de Ciências Biológicas da Universidade Tecnológica Federal do Paraná, Campus Universitário, CEP 85660-000, Dois Vizinhos (PR), Brasil. danielaaestevan@utfpr.edu.br

3 Biólogo, Departamento de Biologia Animal e Vegetal, Universidade Estadual de Londrina, CEP 86057-970, Londrina (PR), Brasil. rossetto.felipe@gmail.com

4 Engenheiro Florestal, Dr., Professor da Coordenação de Engenharia Florestal, Universidade Tecnológica Federal do Paraná, Campus Universitário, CEP 85660-000, Dois Vizinhos (PR), Brasil. bechara@utfpr.edu.br / mauriciorg@ utfpr.edu.br

Recebido para publicação em 12/03/2016 e aceito em 14/09/2016

Ci. Fl., v. 28, n. 1, jan.- mar., 2018 


\section{ABSTRACT}

Due to the increasing environmental degradation, the understanding of how reforestation and other restoration techniques function became fundamental to base the reestablishment of degraded ecosystems. The aim of this study was to perform a vegetation survey in a restoration site through three different techniques. The study site has 7.2 ha in a subtropical forest in Dois Vizinhos, Paraná state, Brazil and it is approximately $20 \mathrm{~m}$ from a secondary forest fragment. The experiment consisted of 12 plots with three treatments $\left(T_{1-3}\right)$ randomly distributed in four blocks. $T_{1}$ : natural regeneration through passive restoration. $T_{2}$ : set of nucleation techniques. $T_{3}$ : seedlings planted through filling and diversity lines $(3 \times 2 \mathrm{~m})$. For sampling purposes, each plot was divided into 24 subplots. In order to evaluate plant presence, two years and eight months after the treatments implementation, a survey was conducted in three random subplots for each plot, where all plants taller than $50 \mathrm{~cm}$ were identified and quantified. Native species richness was higher than $87 \%$ in all treatments, whereas the presence of herbaceous species was higher at passive restoration and woody species at plantation. The most common dispersal syndromes were anemochory and zoochory. By comparing planted and regenerated individuals for the three treatments, the plantation technique had the highest Shannon diversity index (3.017), with 148 individuals and 53 species sampled. However, there was no statistically significant difference. This higher diversity is related to the larger number of species planted, but it is still not facilitating regeneration species in its understory at this age. Considering only regenerated individuals, nucleation showed the highest Shannon diversity index (2.078) with a total of 908 individuals of 40 species, however, it wasn't statistically significant different of passive restoration (Shannon $=1.965$ ) which had 2,018 individuals of 40 species. The most floristic similar treatments were passive restoration and nucleation. Nucleation facilitated species natural regeneration (17 planted and 40 regenerated species) while passive restoration showed high resilience, therefore the use of passive restoration can be considered an effective alternative since its results did not differ statistically from nucleation, and the main advantage of this technique is the reduced cost.

Keywords: nucleation techniques; filling and diversity lines; ecological restoration.

\section{INTRODUÇÃO}

No Brasil, ainda são degradados anualmente aproximadamente $6 \%$ da cobertura vegetal do bioma Mata Atlântica, sendo que o estado do Paraná ocupa o $4^{\circ}$ lugar no ranking de desmatamento (INSTITUTO NACIONAL DE PESQUISAS ESPACIAIS, 2014). A restauração de um ecossistema deve possuir como objetivo o reestabelecimento não somente da estrutura vegetal, mas também da ecologia destas comunidades, de uma forma que facilite a recuperação natural destes ecossistemas (ENGEL; PARROTA, 2003; GOMEZAPARICIO et al., 2004). Ações restauradoras devem estar ligadas desde a remoção de fatores de perturbação e isolamento da área para que a regeneração natural (AIDE et al., 2000; WADT, 2003) seja estimulada restauração passiva - até a interferência do homem através de técnicas para acelerar e influenciar a trajetória da sucessão natural do local - restauração ativa.

Há diversos níveis de intervenção em projetos de restauração ativa: desde níveis mais intermediários tais como o uso de técnicas de irradiação de diversidade através da nucleação (REIS; BECHARA; TRES, 2010; CORBIN; HOLL, 2012; BOANARES; AZEVEDO, 2014; REIS et al., 2014; VOGEL; CAMPOS; BECHARA, 2015), até níveis mais elevados como o plantio de alta diversidade de espécies arbóreas em área total sob linhas de recobrimento e diversidade (RODRIGUES et al., 2009; 2011; BRANCALION; RODRIGUES; GANDOLFI, 2015). Estes projetos estão sendo cada vez mais comuns devido à procura por resultados imediatos, sendo que em relação à restauração passiva, é demandado um tempo maior para que os resultados sejam alcançados. Neste contexto, há uma discussão, baseada em uma série de exemplos em que a restauração passiva deu certo, através dos quais se questiona se a restauração ativa é realmente necessária em todos os casos, visto que esta demanda maior custo, que poderia ser evitado em áreas em que a restauração passiva seria suficiente (HOLL; AIDE, 2011). A estrutura da vegetação restaurada utilizando a restauração ativa e utilizando a restauração passiva pode ser muito distinta. Esta diferença estrutural pode influenciar diversos componentes do ecossistema como, por exemplo, a qualidade do habitat para animais e até a disponibilidade de alimento (MORRISON; LINDELL, 2011). 
É importante ressaltar que uma das metas do governo brasileiro com o intuito de reduzir a emissão de gases de efeito estufa é recuperar 15 milhões de ha de áreas degradadas por pastagens até 2020 (BRASIL, 2012). A recuperação apropriada destas áreas está intimamente ligada à escolha de técnicas adequadas e tais escolhas dependem de estudos que possam auxiliar e informar aos restauradores sobre qual direção seguir em seus projetos de restauração. Neste sentido, este trabalho teve como objetivo avaliar as potencialidades de três diferentes métodos para restauração florestal, discutidos em Bechara et al. (2016): 1) Restauração passiva; 2) Nucleação; e 3) Plantio em linhas de recobrimento e diversidade através de um levantamento de vegetação aos 2 anos e 8 meses de idade no sul do Brasil.

\section{MATERIAL E MÉTODOS}

O local de estudo possui 7,2 ha e está situado no município de Dois Vizinhos, estado do Paraná, sul do Brasil, dentro da fazenda experimental da Universidade Tecnológica Federal do Paraná (25⒋ '44"$25^{\circ} 41^{\prime} 49^{\prime \prime}$ S e $53^{\circ} 06^{\prime} 23^{\prime \prime}-53^{\circ} 06^{\prime} 07^{\prime}$ 'W) (Figura 1). A altitude varia entre 475 e $510 \mathrm{~m}$, presente em um clima subtropical úmido (Cfa) em um ecótono de Floresta Ombrófila Mista com Floresta Estacional Semidecidual. Esta área encontra-se a aproximadamente 20 metros de um fragmento de floresta secundária e anteriormente foi utilizada para cultivo de culturas anuais e pastagens sendo inteiramente roçada (out./2010), quando foi isolada dos fatores de perturbação e os tratamentos foram implantados.

O experimento foi delineado através de 12 parcelas de $40 \times 54 \mathrm{~m}\left(2.160 \mathrm{~m}^{2}\right)$ que foram distribuídas em quatro blocos casualizados com três tratamentos $\left(T_{1-3}\right)$, discorridos a seguir. $T_{1}$ : regeneração natural através da restauração passiva. $\mathrm{T}_{2}$ : conjunto de técnicas de nucleação adaptadas de Reis, Bechara e Tres (2010), em que foram implantados em cada parcela: dois poleiros artificiais para avifauna; seis abrigos artificiais para fauna terrestre, construídos através de pilhas de um estéreo de madeira; plantio de placas de mudas procedentes de banco e chuva de sementes (seis placas de banco e seis placas de chuva) de floresta em estágio médio de sucessão; plantio de árvores em 24 ilhotas de cinco mudas, sob espaçamento 1 x $1 \mathrm{~m}$ (em forma cruz), sendo as quatro plantas laterais espécies sombreadoras (12 pioneiras) e a planta central foi sombreada (24 não pioneiras) totalizando 36 espécies nativas; 24 núcleos de 3 x $4 \mathrm{~m}$ com cobertura de feijão-guandu (Cajanus cajan (L.) Huth); e plantio de bromélias (Bromelia antiacantha Bertol.) em seis ilhotas de cinco mudas, sob espaçamento $0,5 \times 0,5 \mathrm{~m}$ (em forma cruz). O conjunto de técnicas foi inserido em faixas de $3 \times 40 \mathrm{~m}$ espaçadas por seis metros entre faixas. $\mathrm{T}_{3}$ : plantio de mudas em área total de acordo com o método utilizado por Rodrigues et al. (2009), em espaçamento 3 x 2 m totalizando 360 mudas por parcela, através de linhas de recobrimento e diversidade, nas quais foram plantadas, alternadamente dentro da linha, árvores de recobrimento (10 espécies, sendo 18 mudas por espécie) e árvores de diversidade (60 espécies, sendo três mudas por espécie), totalizando 70 espécies nativas.

A área total de plantio em linhas e somente as faixas de nucleação ( $1 / 3$ da área da parcela) receberam práticas silviculturais usuais, tais como adubação, controle de formigas, roçadas e capinas químicas. A coleta de dados foi realizada após dois anos e oito meses da implantação dos tratamentos (jun./2013), quando foi feito um levantamento em três subparcelas de 9 x $10 \mathrm{~m}$ aleatórias para cada parcela.

Foram identificadas e quantificadas todas as plantas maiores que $50 \mathrm{~cm}$ de altura, exceto gramíneas, totalizando uma área amostral de $3.240 \mathrm{~m}^{2}$. As espécies foram classificadas quanto ao hábito (herbácea e lenhosa), forma de dispersão (anemocórica, autocórica e zoocórica), segundo a classificação de Pijl (1982), e origem (nativa e exótica). Obteve-se então o número médio de indivíduos $(\mathrm{Ni})$, a riqueza média de espécies (S) e calcularam-se os parâmetros fitossociológicos de densidade absoluta e relativa e frequência absoluta e relativa, assim como o índice de diversidade de Shannon e índice de equabilidade de Pielou através da utilização do índice médio por parcela em cada tratamento, posteriormente, os índices foram submetidos a uma ANOVA de efeitos principais e as médias dos tratamentos foram comparadas através do teste de Tukey, a 5\%, utilizando o software R 3.3.0®. Foi também calculado o índice de similaridade de Jaccard através do software Excel®. 

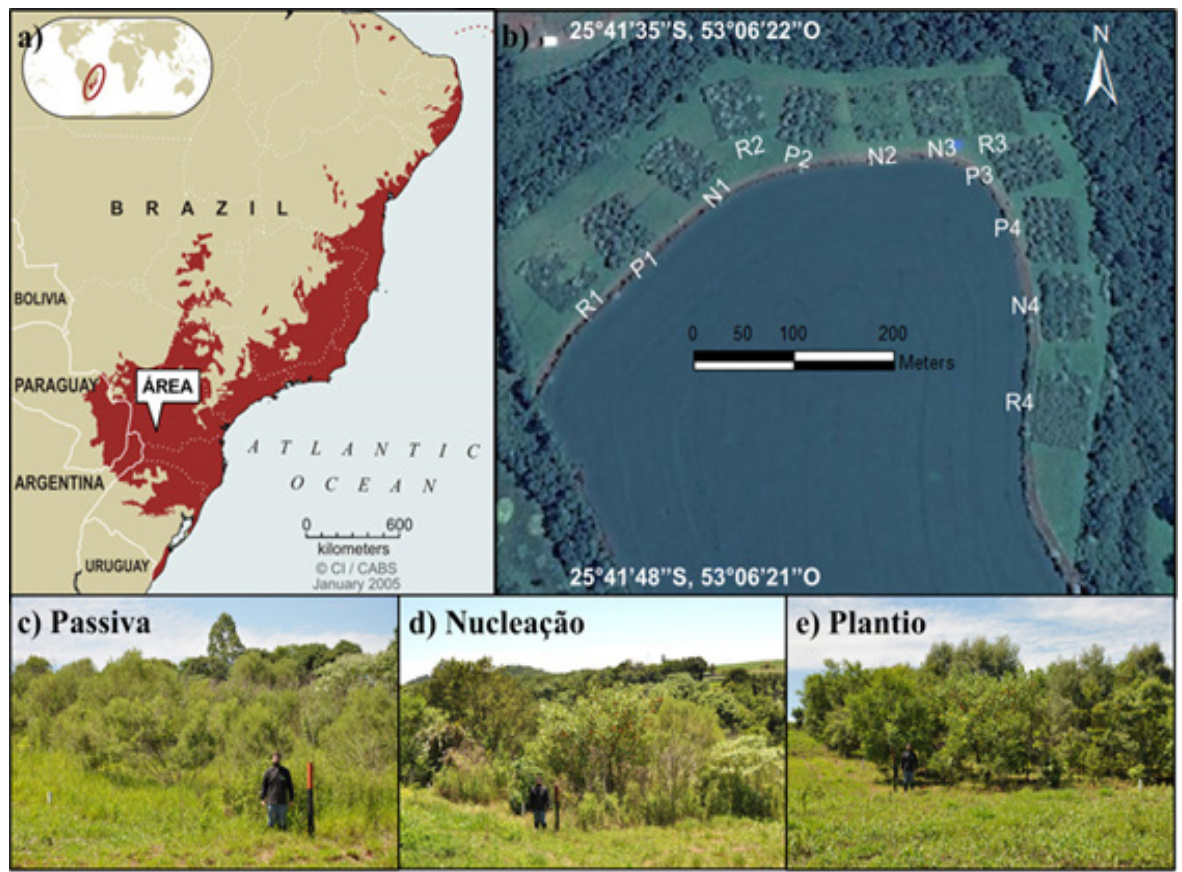

FIGURA 1: a) Localização da área em processo de restauração na fazenda da UTFPR - DV. Fonte: CI/CABS. b) vista aérea da área de estudo, em que: $\mathrm{R}=$ Restauração passiva; $\mathrm{N}=$ Nucleação; $\mathrm{P}=$ Plantio. Fonte: Google Earth. Fotos das diferentes técnicas de restauração aos três anos e dois meses de idade: c) Passiva; d) Nucleação; e e) Plantio.

FIGURE 1: a) Location of the study site in restoration process at UTFPR - DV farm. Source: CI/CABS. b) Study site aerial view where: $\mathrm{R}=$ Passive restoration; $\mathrm{N}=$ Nucleation; $\mathrm{P}=$ Plantation. Source: Google Earth. Pictures of the different restoration techniques at the age of three years and two months: c) Passive; d) Nucleation and e) Plantation.

\section{RESULTADOS E DISCUSSÃO}

Em todos os tratamentos, aproximadamente 95\% das espécies são nativas (Figura 2a), tendo em vista a composição de espécies plantadas e regenerantes. $\mathrm{O}$ tratamento plantio em linhas de recobrimento e diversidade (PL) apresentou a maior porcentagem de espécies nativas $(98,1 \%)$, seguido da nucleação (NU) com $91,2 \%$ e da restauração passiva (PA) com 87,5\%. A riqueza de espécies exóticas foi baixa, sendo que o maior número delas (quatro) está na PA. A riqueza de espécies herbáceas (Figura 2b), característica de uma área em estágio inicial de sucessão, na PA e na NU foi similar (26 e 24 espécies), enquanto no PL foi menor (oito espécies). Em relação às espécies lenhosas, observou-se uma relação inversamente proporcional entre os tratamentos PA e PL, em que a PA obteve a maior riqueza de espécies herbáceas (26) e a menor de espécies lenhosas (13) enquanto o PL apresentou a maior riqueza de espécies lenhosas (45) e a menor de espécies herbáceas (8) (Figura 2b). A NU exibiu uma proporção muito similar entre ambas as formas de vida (24 espécies herbáceas e 31 lenhosas), porém, com uma predominância de espécies lenhosas. A predominância de espécies lenhosas no PL ocorreu provavelmente pela grande quantidade de espécies arbóreas implantadas na área e operações de roçagem e capina química em área total eliminando espécies herbáceas.

$\mathrm{Na} \mathrm{NU}$ foi encontrado o maior número de espécies de dispersão anemocórica (23), seguido da PA (19) e PL (13) (Figura 2c). A dispersão autocórica foi igual entre os tratamentos PA e NU (nove espécies). Já o PL apresentou o maior número de espécies autocóricas (14). A zoocoria foi mais comum no tratamento PL (26 espécies), seguido da NU (22), enquanto a PA obteve o menor número de espécies zoocóricas (11). As formas de dispersão com maior número de espécies foram: anemocoria e a zoocoria; a anemocoria é uma síndrome de dispersão mais comum em locais secos, havendo uma relação indiretamente proporcional com o aumento da umidade e a ocorrência de espécies dispersas pelo vento (HOWE; SMALLWOOD, 1982). Uma maior proporção de plantas na maioria das comunidades vegetais possui dispersão zoocórica (HOWE; 
SMALLWOOD, 1982), o que pode explicar o maior número de espécies zoocóricas neste estudo.

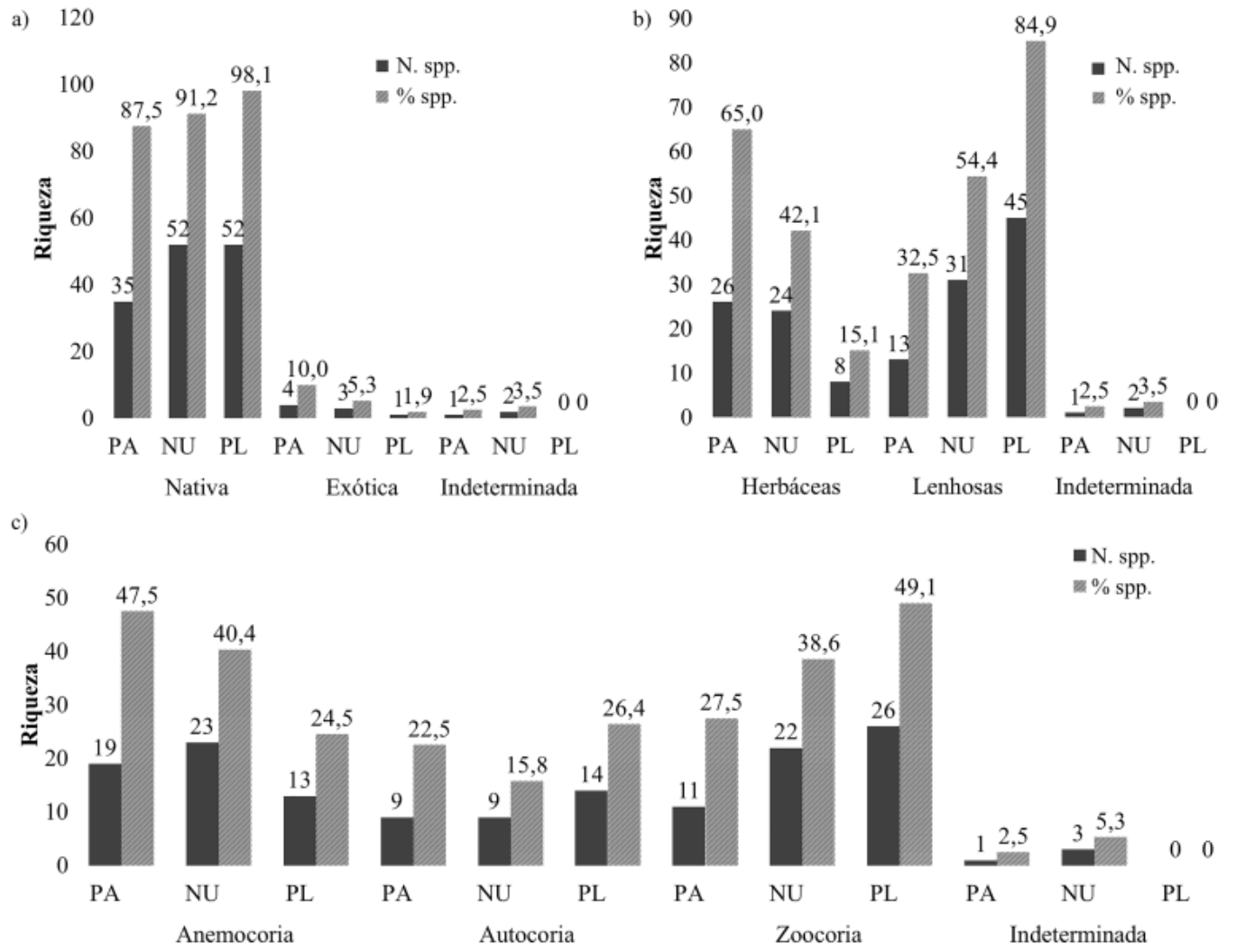

FIGURA 2: Comparação entre tratamentos através de diferentes parâmetros. PA = Restauração passiva, NU = Nucleação, e PL = Plantio em linhas de recobrimento e diversidade.

FIGURE 2: Comparison among treatments throughout different parameters. PA $=$ Passive restoration, $\mathrm{NU}=$ Nucleation, and PL $=$ Coverage and diversity plantation lines.

A densidade de indivíduos nativos (Figura 3a) foi maior no tratamento PA (18.601 ind.ha-1), devido ao grande número de indivíduos encontrados neste tratamento (Tabela 1) em comparação aos outros (Tabelas 2 e 3), seguido pelo tratamento NU (8.351 ind.ha ${ }^{-1}$ ) e o tratamento PL (1.361 ind.ha ( $^{-1}$ ). Em relação aos indivíduos exóticos, o tratamento que apresentou a maior densidade foi a NU (4.055 ind.ha $\left.{ }^{-1}\right)$, e esta foi bem maior do que os outros dois tratamentos $\left(\mathrm{PA}=74\right.$ ind ha $^{-1} \mathrm{e} \mathrm{PL}=9$ ind.ha $\left.^{-1}\right)$. Esta grande densidade de indivíduos exóticos no tratamento NU possivelmente se deve ao fato de que a espécie exótica Cajanus cajan que foi plantada na área como parte do experimento, foi semeada em 24 núcleos por parcela, originando um grande número de indivíduos. Esta espécie foi introduzida para promover a melhoria da qualidade do solo, por sua capacidade de fixação de nitrogênio e por ser uma espécie que possui um ciclo de vida curto. A PA apresentou a maior densidade de indivíduos herbáceos (14.962 ind.ha-1) seguida da NU (6.787 ind.ha ${ }^{-1}$ ) (Figura 3b). Em relação às espécies lenhosas, o tratamento de maior densidade foi a NU (5.620 ind.ha ${ }^{-1}$ ) seguido da PA (3.712 ind.ha $\left.{ }^{-1}\right)$ e do PL (1.074 ind.ha $\left.{ }^{-1}\right)$. Em relação às síndromes de dispersão (Figura 3c), a PA apresentou a maior densidade de indivíduos anemocóricos (17.777 ind.ha $\left.{ }^{-1}\right)$ seguida da NU (6.879 ind.ha $\left.{ }^{-1}\right)$, e por último o PL (388 ind.ha $\left.{ }^{-1}\right)$. Quanto à dispersão autocórica, a NU obteve a maior densidade (4.592 ind.ha $\left.{ }^{-1}\right)$ e foi maior que os outros dois tratamentos $\left(\mathrm{PA}=277\right.$ ind.ha $^{-1}$ e PL $=435$ ind. $\left.\mathrm{ha}^{-1}\right)$. A nucleação apresentou a maior densidade de indivíduos autocóricos provavelmente pela espécie Cajanus cajan novamente, que se apresentou em grande densidade, e possui dispersão autocórica. Quanto à dispersão zoocórica, todos os tratamentos apresentaram valores similares $\left(\mathrm{PA}=620\right.$ ind.ha- ${ }^{-1}, \mathrm{NU}=879$ ind. ha $^{-1}$ e PL $=546$ ind.ha ${ }^{-1}$ ), sendo que a NU apresentou a maior densidade. 

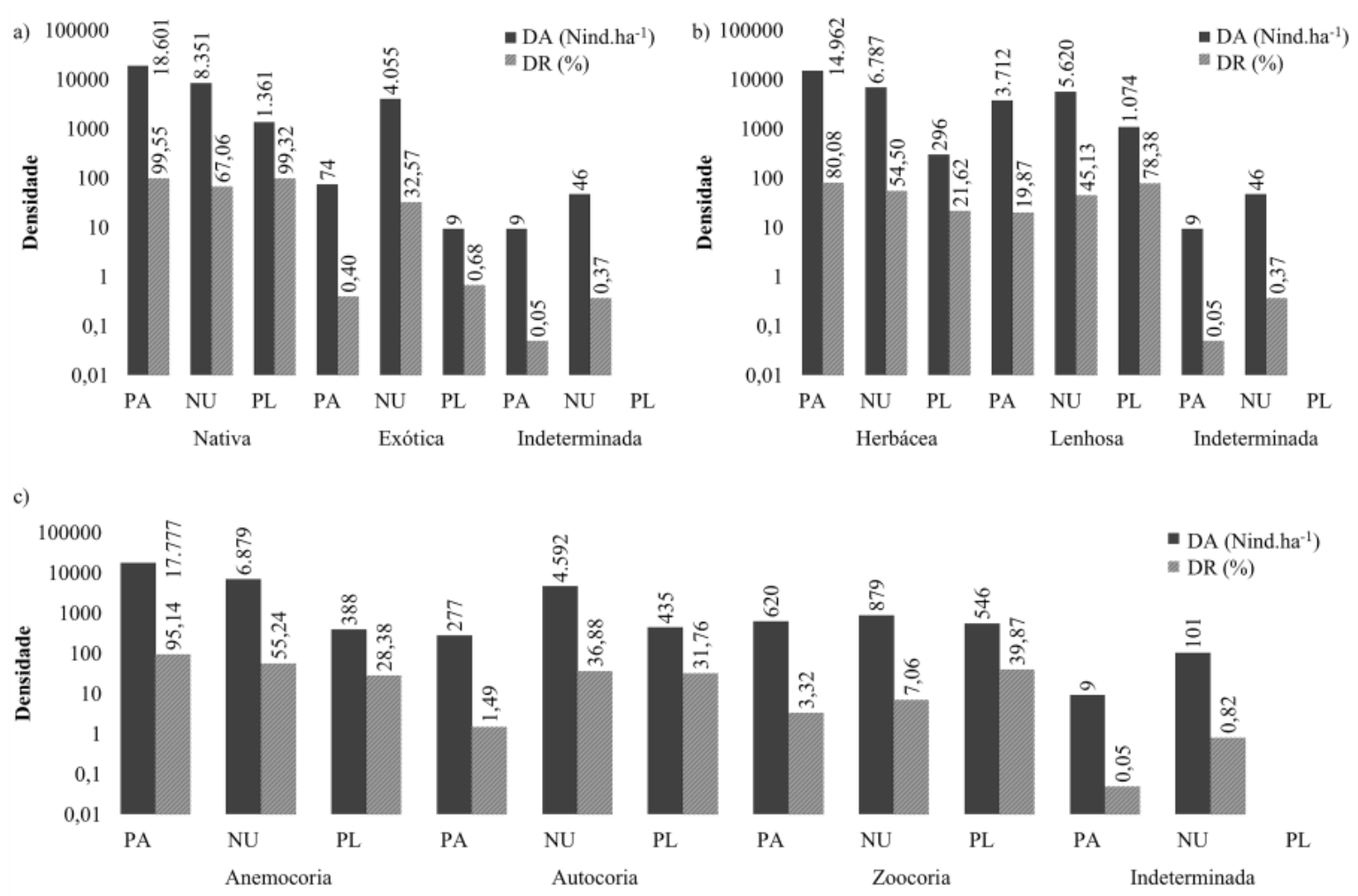

FIGURA 3: Comparação entre tratamentos através de diferentes parâmetros. DA = densidade absoluta (ind.ha ${ }^{-1}$ ), $\mathrm{DR}=$ densidade relativa (\%), PA = Restauração passiva, $\mathrm{NU}=$ Nucleação, e $\mathrm{PL}=$ Plantio em linhas de recobrimento e diversidade.

FIGURE 3: Comparison among treatments throughout different parameters. $\mathrm{DA}=$ absolute density $\left(\right.$ ind.ha $\left.^{-1}\right), \mathrm{DR}=$ relative density $(\%), \mathrm{PA}=$ Passive restoration, $\mathrm{NU}=$ Nucleation, $\mathrm{PL}=$ Coverage and diversity plantation lines.

No tratamento PA foram identificadas 20 famílias botânicas (Figura 4). Dentre estas famílias, a que apresentou maior número de espécies foi Asteraceae (Figura 4a). A segunda família com maior número de espécies foi Solanaceae com quatro espécies distintas. É importante também destacar a terceira família de maior ocorrência no tratamento PA por se tratar de uma família composta geralmente por lianas, Convolvulaceae. No tratamento NU foram amostradas 28 famílias distintas, dentre as quais, a que apresentou o maior número de espécies foi Asteraceae (Figura 4b), da mesma forma que na PA. A segunda família com maior número de espécies foi Solanaceae, novamente da mesma forma que na restauração passiva, com cinco espécies distintas. Já a terceira família com maior número de espécies na nucleação foi a família Fabaceae, com quatro espécies distintas. Através de uma análise florística e visual da Figura 4, denota-se certo grau de similaridade entre famílias botânicas na PA e na NU, a qual enriqueceu a comunidade com algumas famílias adicionais. Já no tratamento PL, foram identificadas 23 famílias botânicas distintas, sendo a família com maior número de espécies amostradas Fabaceae (Figura 4c). As segundas famílias com o maior número de espécies foram: Asteraceae e Myrtaceae.

O número de famílias amostradas neste estudo foi similar ao encontrado em outros estudos realizados em Floresta Ombrófila Mista (LIEBSCH; ACRA, 2004; SOUZA et al., 2012; FIORENTIN et al., 2015) cujo critério de inclusão foi o mesmo ou similar (acima de $30 \mathrm{~cm}$ ). Foram encontradas de 23 a 32 famílias distintas. Em relação às famílias de maior ocorrência, alguns estudos (LIEBSCH; ACRA, 2004; SOUZA et al., 2012) também apresentaram a família Asteraceae entre as duas famílias de maior ocorrência.

No tratamento PA, foi amostrado um total de 2.018 indivíduos pertencentes a 40 espécies (Tabela 1). A densidade absoluta de indivíduos foi de $18.685,19$ um valor similar ao encontrado em outro estudo (23.040 ind.ha-1) em Floresta Ombrófila Mista (SOUZA et al., 2012), avaliando somente o estrato 
regenerante, indicando que esta densidade apresenta-se de acordo com as características da regeneração natural de uma floresta clímax. As cinco espécies de maior densidade são todas anemocóricas e em sua maioria espécies herbáceas. Austroeupatorium inulifolium (Kunth) R.M.King \& H.Rob. (mata-pasto), espécie nativa com forma de vida herbácea, foi a espécie de maior densidade $(28,94 \%)$. A segunda espécie de maior densidade $(25,22 \%)$ foi Mikania cordifolia (L.f.) Willd (erva-de-cobra), uma liana nativa. Sida rhombifolia (guanxuma) foi a terceira espécie de maior densidade $(9,61 \%)$, sendo esta uma espécie nativa e herbácea. Senecio brasiliensis (Spreng.) Less. (maria-mole) foi a quarta espécie de maior densidade (8,92\%). Trata-se de uma espécie herbácea e nativa. A quinta espécie de maior densidade $(7,73 \%)$ foi Vernonanthura tweediana (Baker) H. Rob., conhecida como assa-peixe, esta espécie é nativa, e lenhosa. Todas as espécies de maior densidade são ervas, arbustos e cipós, espécies características de estágios sucessionais iniciais.

a)

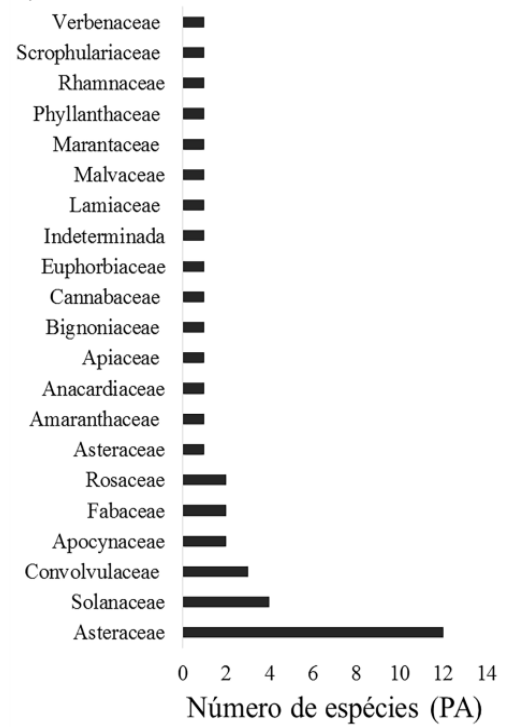

b)

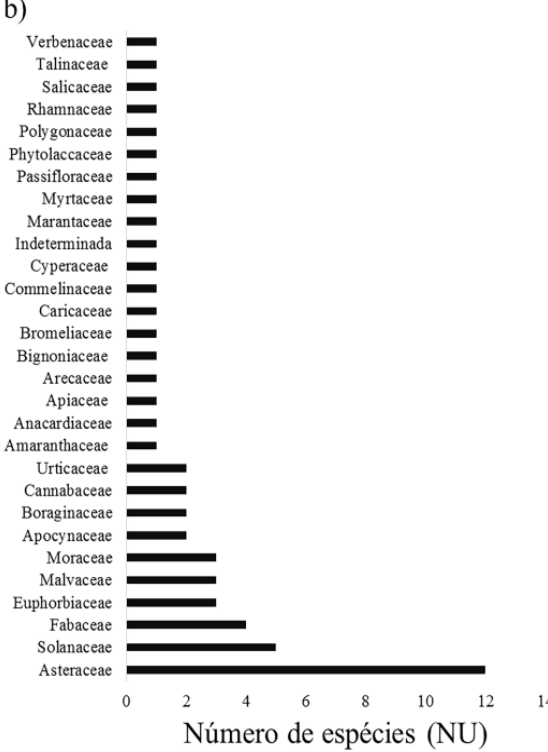

c)

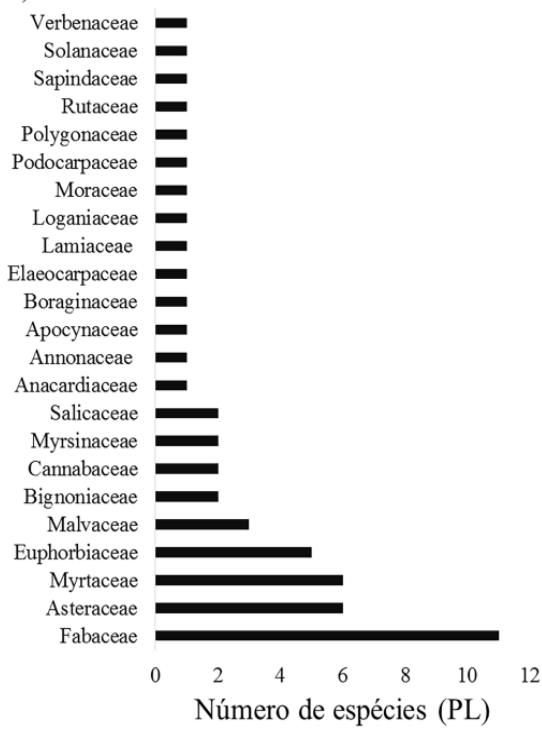

FIGURA 4: a) Lista das famílias botânicas e número de espécies por família amostradas no tratamento de Restauração passiva (PA). b) Lista das famílias botânicas e número de espécies por família amostradas no tratamento de Nucleação (NU). c) Lista das famílias botânicas e número de espécies por família amostradas no tratamento de Plantio em linhas de recobrimento e diversidade (PL).

FIGURE 4: a) Botanical families list and species number for each family sampled in the passive restoration treatment (PA). b) List of botanical families and species number for each family sampled in the Nucleation treatment (NU). c) List of botanical families and species number for each family sampled in the coverage and diversity plantation lines treatment $(\mathrm{PL})$.

TABELA 1: Espécies amostradas em área em recuperação através da Restauração Passiva.

TABLE 1: Sampled species in restoration site through Passive restoration.

\begin{tabular}{|c|c|c|c|c|c|c|c|c|c|}
\hline Espécies & OR & $\mathrm{FV}$ & SDP & $\mathrm{P} / \mathrm{R}$ & $\mathrm{Ni}$ & Dai & Dri & Fai & Fri \\
\hline $\begin{array}{l}\text { Austroeupatorium inulifolium (Kunth) } \\
\text { R.M.King \& H.Rob. }\end{array}$ & $\mathrm{N}$ & $\mathrm{H}$ & AN & $\mathrm{R}$ & 584 & 5407,41 & 28,94 & 100,00 & 8,82 \\
\hline Mikania cordifolia (L.f.) Willd & $\mathrm{N}$ & $\mathrm{H}$ & $\mathrm{AN}$ & $\mathrm{R}$ & 509 & 4712,96 & 25,22 & 91,67 & 8,09 \\
\hline Sida rhombifolia L. & $\mathrm{N}$ & $\mathrm{H}$ & AN & $\mathrm{R}$ & 194 & 1796,30 & 9,61 & 83,33 & 7,35 \\
\hline
\end{tabular}


TABELA 1: Continua...

TABLE 2: Continua...

\begin{tabular}{|c|c|c|c|c|c|c|c|c|c|}
\hline Espécies & OR & $\mathrm{FV}$ & SDP & $\mathrm{P} / \mathrm{R}$ & $\mathrm{Ni}$ & Dai & Dri & Fai & Fri \\
\hline Senecio brasiliensis (Spreng.) Less. & $\mathrm{N}$ & $\mathrm{H}$ & AN & $\mathrm{R}$ & 180 & 1666,67 & 8,92 & 66,67 & 5,88 \\
\hline Vernonanthura tweediana (Baker) H. Rob. & $\mathrm{N}$ & $\mathrm{L}$ & AN & $\mathrm{R}$ & 156 & 1444,44 & 7,73 & 100,00 & 8,82 \\
\hline $\begin{array}{l}\text { Chromolaena maximilianii (generic } \\
\text { SCHRADER ex DC.) R.M. King \& H. Rob. }\end{array}$ & $\mathrm{N}$ & $\mathrm{L}$ & AN & $\mathrm{R}$ & 95 & 879,63 & 4,71 & 66,67 & 5,88 \\
\hline Baccharis dracunculifolia DC. & $\mathrm{N}$ & $\mathrm{L}$ & AN & $\mathrm{R}$ & 88 & 814,81 & 4,36 & 75,00 & 6,62 \\
\hline Baccharis oxyodonta DC. & $\mathrm{N}$ & $\mathrm{H}$ & AN & $\mathrm{R}$ & 67 & 620,37 & 3,32 & 83,33 & 7,35 \\
\hline Amphilophium crucigerum (L.) L. Lohmann & $\mathrm{N}$ & $\mathrm{H}$ & AN & $\mathrm{R}$ & 30 & 277,78 & 1,49 & 50,00 & 4,41 \\
\hline Solanum guaraniticum A.St.-Hil. & $\mathrm{N}$ & $\mathrm{L}$ & $\mathrm{ZO}$ & $\mathrm{R}$ & 26 & 240,74 & 1,29 & 25,00 & 2,21 \\
\hline Euphorbia heterophylla L. & $\mathrm{N}$ & $\mathrm{H}$ & $\mathrm{AU}$ & $\mathrm{R}$ & 21 & 194,44 & 1,04 & 33,33 & 2,94 \\
\hline Schinus terebinthifolius Raddi. & $\mathrm{N}$ & $\mathrm{L}$ & $\mathrm{ZO}$ & $\mathrm{R}$ & 11 & 101,85 & 0,55 & 50,00 & 4,41 \\
\hline Solanum mauritianum Scop. & $\mathrm{N}$ & $\mathrm{L}$ & $\mathrm{ZO}$ & $\mathrm{R}$ & 10 & 92,59 & 0,50 & 58,33 & 5,15 \\
\hline Trema micranta (L.) Blume & $\mathrm{N}$ & $\mathrm{L}$ & $\mathrm{ZO}$ & $\mathrm{R}$ & 7 & 64,81 & 0,35 & 16,67 & 1,47 \\
\hline Achyrocline satureioides (Lam.) DC. & $\mathrm{N}$ & $\mathrm{H}$ & AN & $\mathrm{R}$ & 4 & 37,04 & 0,20 & 8,33 & 0,74 \\
\hline Rubus rosifolius Sm. & $\mathrm{E}$ & $\mathrm{H}$ & $\mathrm{ZO}$ & $\mathrm{R}$ & 4 & 37,04 & 0,20 & 8,33 & 0,74 \\
\hline Asclepias curassavica $\mathrm{L}$. & $\mathrm{N}$ & $\mathrm{H}$ & AN & $\mathrm{R}$ & 3 & 27,78 & 0,15 & 8,33 & 0,74 \\
\hline Pterocaulon alopecuroides (Lam.) DC. & $\mathrm{N}$ & $\mathrm{H}$ & AN & $\mathrm{R}$ & 3 & 27,78 & 0,15 & 16,67 & 1,47 \\
\hline Hovenia dulcis Thunb. & $\mathrm{E}$ & $\mathrm{L}$ & $\mathrm{ZO}$ & $\mathrm{R}$ & 2 & 18,52 & 0,10 & 8,33 & 0,74 \\
\hline Ipomoea quamoclit $\mathrm{L}$. & $\mathrm{N}$ & $\mathrm{H}$ & $\mathrm{AU}$ & $\mathrm{R}$ & 2 & 18,52 & 0,10 & 8,33 & 0,74 \\
\hline Maranta sobolifera L. Andersson & $\mathrm{N}$ & $\mathrm{H}$ & $\mathrm{ZO}$ & $\mathrm{R}$ & 2 & 18,52 & 0,10 & 16,67 & 1,47 \\
\hline Solanum pseudoquina A.St.-Hil. & $\mathrm{N}$ & $\mathrm{L}$ & $\mathrm{ZO}$ & $\mathrm{R}$ & 2 & 18,52 & 0,10 & 8,33 & 0,74 \\
\hline Buddleja stachyoides Cham. \& Schltdl. & $\mathrm{N}$ & $\mathrm{L}$ & $\mathrm{AU}$ & $\mathrm{R}$ & 1 & 9,26 & 0,05 & 8,33 & 0,74 \\
\hline $\begin{array}{l}\text { Ciclospermum leptophyllum (Pers.) Sprague } \\
\text { var. leptophyllum }\end{array}$ & $\mathrm{N}$ & $\mathrm{H}$ & $\mathrm{AU}$ & $\mathrm{R}$ & 1 & 9,26 & 0,05 & 8,33 & 0,74 \\
\hline Convolvulus crenatifolius Ruiz \& Pav. & $\mathrm{N}$ & $\mathrm{H}$ & $\mathrm{AU}$ & $\mathrm{R}$ & 1 & 9,26 & 0,05 & 8,33 & 0,74 \\
\hline Conyza bonariensis (L.) Cronquist & $\mathrm{N}$ & $\mathrm{H}$ & AN & $\mathrm{R}$ & 1 & 9,26 & 0,05 & 8,33 & 0,74 \\
\hline $\begin{array}{l}\text { Dysphania ambrosioides (L.) Mosyakin \& } \\
\text { Clemants }\end{array}$ & $\mathrm{N}$ & $\mathrm{H}$ & AN & $\mathrm{R}$ & 1 & 9,26 & 0,05 & 8,33 & 0,74 \\
\hline Ipomoea indivisa (Vell.) Hallier f. & $\mathrm{N}$ & $\mathrm{H}$ & $\mathrm{AU}$ & $\mathrm{R}$ & 1 & 9,26 & 0,05 & 8,33 & 0,74 \\
\hline Leonurus japonicus Houtt. & $\mathrm{E}$ & $\mathrm{H}$ & $\mathrm{AU}$ & $\mathrm{R}$ & 1 & 9,26 & 0,05 & 8,33 & 0,74 \\
\hline Lepidaploa balansae (Hieron.) H. Rob. & $\mathrm{N}$ & $\mathrm{H}$ & AN & $\mathrm{R}$ & 1 & 9,26 & 0,05 & 8,33 & 0,74 \\
\hline Lepidaploa salzmannii (DC.) H.Rob. & $\mathrm{N}$ & $\mathrm{L}$ & AN & $\mathrm{R}$ & 1 & 9,26 & 0,05 & 8,33 & 0,74 \\
\hline Parapiptadenia rigida (Benth.) Brenan & $\mathrm{N}$ & $\mathrm{L}$ & AN & $\mathrm{R}$ & 1 & 9,26 & 0,05 & 8,33 & 0,74 \\
\hline Phyllanthus tenellus Roxb. & $\mathrm{N}$ & $\mathrm{H}$ & $\mathrm{AU}$ & $\mathrm{R}$ & 1 & 9,26 & 0,05 & 8,33 & 0,74 \\
\hline Prestonia coalita (Vell.) Woodson & $\mathrm{N}$ & $\mathrm{H}$ & AN & $\mathrm{R}$ & 1 & 9,26 & 0,05 & 8,33 & 0,74 \\
\hline Prunus sellowii Koehne & $\mathrm{N}$ & $\mathrm{L}$ & $\mathrm{ZO}$ & $\mathrm{R}$ & 1 & 9,26 & 0,05 & 8,33 & 0,74 \\
\hline Solanum americanum Mill. & $\mathrm{N}$ & $\mathrm{H}$ & $\mathrm{ZO}$ & $\mathrm{R}$ & 1 & 9,26 & 0,05 & 8,33 & 0,74 \\
\hline Solidago cf. chilensis Meyen & $\mathrm{N}$ & $\mathrm{H}$ & $\mathrm{AN}$ & $\mathrm{R}$ & 1 & 9,26 & 0,05 & 8,33 & 0,74 \\
\hline Indeterminada 1 & IN & IN & IN & $\mathrm{R}$ & 1 & 9,26 & 0,05 & 8,33 & 0,74 \\
\hline Verbena litoralis Kunth var. litoralis & $\mathrm{N}$ & $\mathrm{H}$ & $\mathrm{ZO}$ & $\mathrm{R}$ & 1 & 9,26 & 0,05 & 8,33 & 0,74 \\
\hline Vicia sativa $\mathrm{L}$. & $\mathrm{E}$ & $\mathrm{H}$ & $\mathrm{AU}$ & $\mathrm{R}$ & 1 & 9,26 & 0,05 & 8,33 & 0,74 \\
\hline Total & & & & & 2018 & 18685,19 & 100,00 & 1133,33 & 100,00 \\
\hline
\end{tabular}

Em que: $\mathrm{OR}=$ origem $(\mathrm{N}=$ nativa, $\mathrm{E}=$ exótica, $\mathrm{IN}=$ indeterminada $), \mathrm{FV}=$ forma de vida $(\mathrm{H}=$ herbácea, $\mathrm{L}=$ lenhosa, $\mathrm{IN}=$ indeterminada $), \mathrm{SDP}=$ síndrome de dispersão $(\mathrm{AN}=$ anemocoria, $\mathrm{AU}=$ autocoria, $\mathrm{ZO}=$ zoocoria, $\mathrm{IN}=$ indeterminada), $\mathrm{P} / \mathrm{R}=$ plantada ou regenerante $(\mathrm{P}=$ plantada, $\mathrm{R}=$ regenerante $), \mathrm{Ni}=$ número de indivíduos, $\mathrm{Dai}=$ densidade absoluta $\left(\right.$ Nind $\left.\mathrm{ha}^{-1}\right)$, Dri $=$ densidade relativa $(\%), \mathrm{Fai}=$ frequência absoluta $(\%)$, Fri = frequência relativa $(\%)$.

Foi amostrado um total de 1.345 indivíduos no tratamento NU, pertencentes a 57 espécies (Tabela 2) 
um número de espécie similar a outro estudo que encontrou 53 espécies levando em consideração o estrato regenerante e 56 espécies tendo em conta o estrato adulto (SOUZA et al., 2012). Este tratamento apresentou um total de 17 espécies plantadas amostradas como parte do tratamento e 40 espécies regenerantes, sendo que os indivíduos germinados e recrutados do banco e chuva de sementes (uma das técnicas de nucleação utilizada) foram considerados como regenerantes. A primeira espécie de maior densidade $(28,40 \%)$ foi Cajanus cajan (feijão-guandu). Trata-se de uma espécie exótica, todavia, por ser uma espécie de ciclo de vida curto, pode ser utilizada em áreas de restauração para melhorar a qualidade do solo.

Pode-se notar que Arachis pintoi Krap. \& Greg. (amendoim-forrageiro) apresentou uma densidade relativamente alta neste tratamento (4,01\%), o que não é um bom indicador, por se tratar de uma espécie exótica utilizada em pastagens anteriormente semeadas no local. Esta espécie formou uma espécie de "tapete" na área a partir da germinação de seu banco de sementes, apresentando comportamento invasor e podendo impedir o desenvolvimento de outras espécies, sendo que sua presença foi observada tanto em áreas sombreadas quanto ao pleno sol.

A segunda espécie de maior densidade $(17,77 \%)$ foi $A$. inulifolium. A terceira espécie de maior ocorrência foi a liana M. cordifolia com $10,33 \%$ da densidade total, seguida da erva Senecio brasiliensis com $9,59 \%$ da densidade e o arbusto $V$. tweediana com 5,2\%.

No tratamento PL foi amostrado um total de 148 indivíduos de 53 espécies distintas. Destas, 44 foram plantadas como parte do tratamento e somente nove foram regenerantes (Tabela 3).

TABELA 2: Espécies amostradas em área em recuperação através da Nucleação.

TABLE 2: Sampled species in restoration site through Nucleation.

\begin{tabular}{|c|c|c|c|c|c|c|c|c|c|}
\hline Espécies & OR & $\mathrm{FV}$ & SDP & $\mathrm{P} / \mathrm{R}$ & $\mathrm{Ni}$ & Dai & Dri & Fai & Fri \\
\hline Cajanus cajan (L.) Huth & $\mathrm{E}$ & $\mathrm{L}$ & $\mathrm{AU}$ & $\mathrm{P}$ & 382 & 3537,04 & 28,40 & 100,00 & 7,36 \\
\hline $\begin{array}{l}\text { Austroeupatorium inulifolium (Kunth) R.M.King \& } \\
\text { H.Rob. }\end{array}$ & $\mathrm{N}$ & $\mathrm{H}$ & $\mathrm{AN}$ & $\mathrm{R}$ & 239 & 2212,96 & 17,77 & 83,33 & 6,13 \\
\hline Mikania cordifolia (L.f.) Willd & $\mathrm{N}$ & $\mathrm{H}$ & AN & $\mathrm{R}$ & 139 & 1287,04 & 10,33 & 75,00 & 5,52 \\
\hline Senecio brasiliensis (Spreng.) Less. & $\mathrm{N}$ & $\mathrm{H}$ & $\mathrm{AN}$ & $\mathrm{R}$ & 129 & 1194,44 & 9,59 & 58,33 & 4,29 \\
\hline Vernonanthura tweediana (Baker) H. Rob. & $\mathrm{N}$ & $\mathrm{L}$ & AN & $\mathrm{R}$ & 70 & 648,15 & 5,20 & 75,00 & 5,52 \\
\hline Sida rhombifolia $\mathrm{L}$. & $\mathrm{N}$ & $\mathrm{H}$ & $\mathrm{AN}$ & $\mathrm{R}$ & 59 & 546,30 & 4,39 & 58,33 & 4,29 \\
\hline Arachis pintoi Krapov. \& W.C. Greg. & $\mathrm{E}$ & $\mathrm{H}$ & $\mathrm{AU}$ & $\mathrm{R}$ & 54 & 500,00 & 4,01 & 25,00 & 1,84 \\
\hline Piptocarpha axillaris (Less.) Baker & $\mathrm{N}$ & $\mathrm{L}$ & AN & $\mathrm{R}$ & 25 & 231,48 & 1,86 & 16,67 & 1,23 \\
\hline Baccharis dracunculifolia DC. & $\mathrm{N}$ & $\mathrm{L}$ & AN & $\mathrm{R}$ & 23 & 212,96 & 1,71 & 83,33 & 6,13 \\
\hline Baccharis oxyodonta DC. & $\mathrm{N}$ & $\mathrm{H}$ & AN & $\mathrm{R}$ & 19 & 175,93 & 1,41 & 58,33 & 4,29 \\
\hline Bromelia antiacantha Bertol. & $\mathrm{N}$ & $\mathrm{H}$ & $\mathrm{ZO}$ & $\mathrm{P}$ & 15 & 138,89 & 1,12 & 16,67 & 1,23 \\
\hline $\begin{array}{l}\text { Chromolaena maximilianii (generic SCHRADER ex } \\
\text { DC.) R.M. King \& H. Rob. }\end{array}$ & $\mathrm{N}$ & $\mathrm{L}$ & AN & $\mathrm{R}$ & 15 & 138,89 & 1,12 & 50,00 & 3,68 \\
\hline Commelina benghalensis L. & $\mathrm{N}$ & $\mathrm{H}$ & $\mathrm{AU}$ & $\mathrm{R}$ & 15 & 138,89 & 1,12 & 25,00 & 1,84 \\
\hline Guazuma ulmifolia Lam. & $\mathrm{N}$ & $\mathrm{L}$ & $\mathrm{ZO}$ & $P$ & 12 & 111,11 & 0,89 & 33,33 & 2,45 \\
\hline Prestonia coalita (Vell.) Woodson & $\mathrm{N}$ & $\mathrm{H}$ & AN & $\mathrm{R}$ & 11 & 101,85 & 0,82 & 8,33 & 0,61 \\
\hline Amphilophium crucigerum (L.) L. Lohmann & $\mathrm{N}$ & $\mathrm{H}$ & AN & $\mathrm{R}$ & 10 & 92,59 & 0,74 & 16,67 & 1,23 \\
\hline Trema micranta (L.) Blume & $\mathrm{N}$ & $\mathrm{L}$ & $\mathrm{ZO}$ & $\mathrm{R}$ & 10 & 92,59 & 0,74 & 16,67 & 1,23 \\
\hline Achyrocline satureioides (Lam.) DC. & $\mathrm{N}$ & $\mathrm{H}$ & AN & $\mathrm{R}$ & 9 & 83,33 & 0,67 & 33,33 & 2,45 \\
\hline Solanum mauritianum Scop. & $\mathrm{N}$ & $\mathrm{L}$ & $\mathrm{ZO}$ & $\mathrm{R}$ & 8 & 74,07 & 0,59 & 58,33 & 4,29 \\
\hline Cecropia pachystachya Trécul & $\mathrm{N}$ & $\mathrm{L}$ & $\mathrm{ZO}$ & $\mathrm{R}$ & 7 & 64,81 & 0,52 & 25,00 & 1,84 \\
\hline Euphorbia heterophylla L. & $\mathrm{N}$ & $\mathrm{H}$ & $\mathrm{AU}$ & $\mathrm{R}$ & 7 & 64,81 & 0,52 & 41,67 & 3,07 \\
\hline Heliotropium transalpinum Vell. & $\mathrm{N}$ & $\mathrm{L}$ & $\mathrm{ZO}$ & $\mathrm{R}$ & 6 & 55,56 & 0,45 & 16,67 & 1,23 \\
\hline Saranthe eichleri Petersen & $\mathrm{N}$ & $\mathrm{H}$ & IN & $\mathrm{R}$ & 6 & 55,56 & 0,45 & 8,33 & 0,61 \\
\hline Schinus terebinthifolius Raddi. & $\mathrm{N}$ & $\mathrm{L}$ & $\mathrm{ZO}$ & $\mathrm{R}$ & 6 & 55,56 & 0,45 & 33,33 & 2,45 \\
\hline Trema micranta (L.) Blume & $\mathrm{N}$ & $\mathrm{L}$ & $\mathrm{ZO}$ & $\mathrm{P}$ & 6 & 55,56 & 0,45 & 25,00 & 1,84 \\
\hline
\end{tabular}


TABELA 2: Continuação...

TABLE 2: Continued...

\begin{tabular}{|c|c|c|c|c|c|c|c|c|c|}
\hline Espécies & FV & SDP & $\mathrm{P} / \mathrm{R}$ & $\mathrm{Ni}$ & & Dai & Dri & Fai & Fri \\
\hline Acalypha brasiliensis Müll.Arg. & $\mathrm{N}$ & $\mathrm{H}$ & AU & $\mathrm{R}$ & 5 & 46,30 & 0,37 & 8,33 & 0,61 \\
\hline Bastardiopsis densiflora (Hook. \& Arn.) Hassl. & $\mathrm{N}$ & $\mathrm{L}$ & $\mathrm{AN}$ & $\mathrm{R}$ & 5 & 46,30 & 0,37 & 8,33 & 0,61 \\
\hline Aegiphyla sp. & $\mathrm{N}$ & $\mathrm{L}$ & $\mathrm{ZO}$ & $\mathrm{P}$ & 4 & 37,04 & 0,30 & 8,33 & 0,61 \\
\hline $\begin{array}{l}\text { Ciclospermum leptophyllum (Pers.) Sprague var. } \\
\text { leptophyllum }\end{array}$ & $\mathrm{N}$ & $\mathrm{H}$ & AU & $\mathrm{R}$ & 4 & 37,04 & 0,30 & 8,33 & 0,61 \\
\hline Cyperus virens Michx. & $\mathrm{N}$ & $\mathrm{H}$ & AN & $\mathrm{R}$ & 4 & 37,04 & 0,30 & 16,67 & 1,23 \\
\hline Indeterminada 2 & IN & IN & $\mathrm{IN}$ & $\mathrm{R}$ & 4 & 37,04 & 0,30 & 8,33 & 0,61 \\
\hline Croton urucurana Baill. & $\mathrm{N}$ & $\mathrm{L}$ & AU & $\mathrm{P}$ & 3 & 27,78 & 0,22 & 8,33 & 0,61 \\
\hline Maclura tinctoria (L.) D.Don ex Steud. & $\mathrm{N}$ & $\mathrm{L}$ & $\mathrm{ZO}$ & $\mathrm{R}$ & 3 & 27,78 & 0,22 & 8,33 & 0,61 \\
\hline Passiflora alata Curtis & $\mathrm{N}$ & $\mathrm{L}$ & $\mathrm{ZO}$ & $\mathrm{P}$ & 3 & 27,78 & 0,22 & 16,67 & 1,23 \\
\hline Cordia americana (L.) Gottschling \& J.S. Mill. & $\mathrm{N}$ & $\mathrm{L}$ & AN & $\mathrm{P}$ & 2 & 18,52 & 0,15 & 16,67 & 1,23 \\
\hline Ficus enormis (Mart. Ex Miq.) Mart & $\mathrm{N}$ & $\mathrm{L}$ & $\mathrm{ZO}$ & $\mathrm{P}$ & 2 & 18,52 & 0,15 & 16,67 & 1,23 \\
\hline Ficus sp. & $\mathrm{N}$ & $\mathrm{L}$ & $\mathrm{ZO}$ & $\mathrm{R}$ & 2 & 18,52 & 0,15 & 16,67 & 1,23 \\
\hline Hovenia dulcis Thunb. & $\mathrm{E}$ & $\mathrm{L}$ & $\mathrm{ZO}$ & $\mathrm{R}$ & 2 & 18,52 & 0,15 & 8,33 & 0,61 \\
\hline Solanum guaraniticum A.St.-Hil. & $\mathrm{N}$ & $\mathrm{L}$ & $\mathrm{ZO}$ & $\mathrm{R}$ & 2 & 18,52 & 0,15 & 16,67 & 1,23 \\
\hline Boehmeria caudata $\mathrm{Sw}$. & $\mathrm{N}$ & $\mathrm{H}$ & AN & $\mathrm{R}$ & 1 & 9,26 & 0,07 & 8,33 & 0,61 \\
\hline Butia capitata (Mart.) Becc. & $\mathrm{N}$ & $\mathrm{L}$ & $\mathrm{ZO}$ & $\mathrm{P}$ & 1 & 9,26 & 0,07 & 8,33 & 0,61 \\
\hline Campomanesia guazumifolia (Cambess.) O. Berg & $\mathrm{N}$ & $\mathrm{L}$ & $\mathrm{ZO}$ & $\mathrm{P}$ & 1 & 9,26 & 0,07 & 8,33 & 0,61 \\
\hline Casearia decandra Jacq. & $\mathrm{N}$ & $\mathrm{L}$ & $\mathrm{ZO}$ & $\mathrm{P}$ & 1 & 9,26 & 0,07 & 8,33 & 0,61 \\
\hline Dysphania ambrosioides (L.) Mosyakin \& Clemants & $\mathrm{N}$ & $\mathrm{H}$ & AN & $\mathrm{R}$ & 1 & 9,26 & 0,07 & 8,33 & 0,61 \\
\hline Jacaratia spinosa (Aubl.) A. DC. & $\mathrm{N}$ & $\mathrm{L}$ & $\mathrm{ZO}$ & $\mathrm{P}$ & 1 & 9,26 & 0,07 & 8,33 & 0,61 \\
\hline Lepidaploa balansae (Hieron.) H. Rob. & $\mathrm{N}$ & $\mathrm{H}$ & AN & $\mathrm{R}$ & 1 & 9,26 & 0,07 & 8,33 & 0,61 \\
\hline Mimosa scabrella Benth. & $\mathrm{N}$ & $\mathrm{L}$ & AU & $\mathrm{P}$ & 1 & 9,26 & 0,07 & 8,33 & 0,61 \\
\hline Piptadenia gonoacantha (Mart.) J.F. Macbr. & $\mathrm{N}$ & $\mathrm{L}$ & AU & $\mathrm{P}$ & 1 & 9,26 & 0,07 & 8,33 & 0,61 \\
\hline Porophyllum ruderale (Jacq.) Cass. & $\mathrm{N}$ & $\mathrm{H}$ & AN & $\mathrm{R}$ & 1 & 9,26 & 0,07 & 8,33 & 0,61 \\
\hline Ruprechtia laxiflora Meisn. & $\mathrm{N}$ & $\mathrm{L}$ & AN & $\mathrm{P}$ & 1 & 9,26 & 0,07 & 8,33 & 0,61 \\
\hline Seguieria americana $\mathrm{L}$. & $\mathrm{N}$ & $\mathrm{L}$ & AN & $\mathrm{R}$ & 1 & 9,26 & 0,07 & 8,33 & 0,61 \\
\hline Solanum americanum Mill. & $\mathrm{N}$ & $\mathrm{H}$ & $\mathrm{ZO}$ & $\mathrm{R}$ & 1 & 9,26 & 0,07 & 8,33 & 0,61 \\
\hline Solanum mauritianum Scop. & $\mathrm{N}$ & $\mathrm{L}$ & $\mathrm{ZO}$ & $\mathrm{P}$ & 1 & 9,26 & 0,07 & 8,33 & 0,61 \\
\hline Solanum sp. & $\mathrm{N}$ & $\mathrm{H}$ & $\mathrm{ZO}$ & $\mathrm{R}$ & 1 & 9,26 & 0,07 & 8,33 & 0,61 \\
\hline Indeterminada 3 & IN & IN & IN & $\mathrm{R}$ & 1 & 9,26 & 0,07 & 8,33 & 0,61 \\
\hline Symphyotrichum squamatum (Spreng.) G.L. Nesom & $\mathrm{N}$ & $\mathrm{H}$ & AN & $\mathrm{R}$ & 1 & 9,26 & 0,07 & 8,33 & 0,61 \\
\hline Talinum paniculatum (Jacq.) Gaertn. & $\mathrm{N}$ & $\mathrm{H}$ & AN & $\mathrm{R}$ & 1 & 9,26 & 0,07 & 8,33 & 0,61 \\
\hline Total & & & & & 1345 & 12453,70 & 100,00 & 1358,33 & 100,00 \\
\hline
\end{tabular}

Em que: $\mathrm{OR}=$ origem $(\mathrm{N}=$ nativa, $\mathrm{E}=$ exótica, $\mathrm{IN}=$ indeterminada $), \mathrm{FV}=$ forma de vida $(\mathrm{H}=$ herbácea, $\mathrm{L}=$ lenhosa, $\mathrm{IN}=$ indeterminada), $\mathrm{SDP}=$ síndrome de dispersão $(\mathrm{AN}=$ anemocoria, $\mathrm{AU}=$ autocoria, $\mathrm{ZO}=$ zoocoria, $\mathrm{IN}=$ indeteminada $), \mathrm{P} / \mathrm{R}=$ plantada ou regenerante $(\mathrm{P}=$ plantada, $\mathrm{R}=$ regenerante $), \mathrm{Ni}=$ número de indivíduos, $\mathrm{Dai}=$ densidade

Trata-se de um pequeno número de indivíduos, principalmente quando comparado ao número de espécies, no entanto, este resultado é esperado devido às manutenções realizadas em área total do plantio durante os três primeiros anos. A espécie de maior densidade foi a regenerante $M$. cordifolia $(10,14 \%)$. A segunda espécie de maior densidade $(8,11 \%)$ foi a plantada Bauhinia forficata Link (pata-de-vaca), espécie nativa e de dispersão autocórica. Piptadenia gonoacantha (Mart.) J.F. Macbr. (pau-jacaré) foi a terceira espécie de maior densidade, assim como a espécie Schinus terebinthifolius Raddi. (aroeira-pimenteira), ambas com 5,41\%, foram plantadas como espécies de recobrimento. A quinta espécie de maior densidade foi a regenerante Bidens pilosa L. (picão-preto), com 3,38\% da densidade total de indivíduos. Esta espécie 
é uma planta anual e herbácea de dispersão epizoocórica.

TABELA 3: Espécies amostradas em área em recuperação através do Plantio em Linhas de Recobrimento e Diversidade.

TABLE 3: Sampled species in restoration site through Coverage and Diversity Plantation lines.

\begin{tabular}{|c|c|c|c|c|c|c|c|c|c|}
\hline Espécies & OR & FV & SDP & $\mathrm{P} / \mathrm{R}$ & $\mathrm{Ni}$ & Dai & Dri & Fai & Fri \\
\hline Mikania cordifolia (L.f.) Willd & $\mathrm{N}$ & $\mathrm{H}$ & $\mathrm{AN}$ & $\mathrm{R}$ & 15 & 138,89 & 10,14 & 16,67 & 1,55 \\
\hline Bauhinia forficata Link & $\mathrm{N}$ & $\mathrm{L}$ & AU & $\mathrm{P}$ & 12 & 111,11 & 8,11 & 16,67 & 1,55 \\
\hline Piptadenia gonoacantha (Mart.) J.F. Macbr. & $\mathrm{N}$ & $\mathrm{L}$ & $\mathrm{AU}$ & $\mathrm{P}$ & 8 & 74,07 & 5,41 & 8,33 & 0,78 \\
\hline Schinus terebinthifolius Raddi. & $\mathrm{N}$ & $\mathrm{L}$ & $\mathrm{ZO}$ & $\mathrm{P}$ & 8 & 74,07 & 5,41 & 33,33 & 3,10 \\
\hline Croton floribundus Spreng. & $\mathrm{N}$ & $\mathrm{L}$ & $\mathrm{AU}$ & $\mathrm{P}$ & 5 & 46,30 & 3,38 & 8,33 & 0,78 \\
\hline Bidens pilosa $\mathrm{L}$. & $\mathrm{N}$ & $\mathrm{H}$ & $\mathrm{ZO}$ & $\mathrm{R}$ & 5 & 46,30 & 3,38 & 8,33 & 0,78 \\
\hline $\begin{array}{l}\text { Cordia americana (L.) Gottschling \& J.S. } \\
\text { Mill. }\end{array}$ & $\mathrm{N}$ & $\mathrm{L}$ & $\mathrm{AN}$ & $\mathrm{P}$ & 4 & 37,04 & 2,70 & 91,67 & 8,53 \\
\hline Gochnatia polymorpha (Less.) Cabrera & $\mathrm{N}$ & $\mathrm{L}$ & $\mathrm{AN}$ & $\mathrm{P}$ & 4 & 37,04 & 2,70 & 8,33 & 0,78 \\
\hline Cassia leptophylla Vogel & $\mathrm{N}$ & $\mathrm{L}$ & $\mathrm{AU}$ & $\mathrm{P}$ & 4 & 37,04 & 2,70 & 16,67 & 1,55 \\
\hline Lonchocarpus sp. & $\mathrm{N}$ & $\mathrm{L}$ & $\mathrm{AU}$ & $\mathrm{P}$ & 4 & 37,04 & 2,70 & 16,67 & 1,55 \\
\hline $\begin{array}{l}\text { Allophylus edulis (A. St.-Hil., A. Juss. \& } \\
\text { Cambess.) Hieron. Ex Niederl. }\end{array}$ & $\mathrm{N}$ & $\mathrm{L}$ & $\mathrm{ZO}$ & $\mathrm{P}$ & 4 & 37,04 & 2,70 & 8,33 & 0,78 \\
\hline Eugenia uniflora $\mathrm{L}$. & $\mathrm{N}$ & $\mathrm{L}$ & $\mathrm{ZO}$ & $\mathrm{P}$ & 4 & 37,04 & 2,70 & 33,33 & 3,10 \\
\hline Guazuma ulmifolia Lam. & $\mathrm{N}$ & $\mathrm{L}$ & $\mathrm{ZO}$ & $\mathrm{P}$ & 4 & 37,04 & 2,70 & 16,67 & 1,55 \\
\hline Senecio brasiliensis (Spreng.) Less. & $\mathrm{N}$ & $\mathrm{H}$ & AN & $\mathrm{R}$ & 3 & 27,78 & 2,03 & 16,67 & 1,55 \\
\hline Sida rhombifolia $\mathrm{L}$. & $\mathrm{N}$ & $\mathrm{H}$ & $\mathrm{AN}$ & $\mathrm{R}$ & 3 & 27,78 & 2,03 & 8,33 & 0,78 \\
\hline Vernonanthura tweediana (Baker) H. Rob. & $\mathrm{N}$ & $\mathrm{L}$ & $\mathrm{AN}$ & $\mathrm{R}$ & 3 & 27,78 & 2,03 & 33,33 & 3,10 \\
\hline Mimosa scabrella Benth. & $\mathrm{N}$ & $\mathrm{L}$ & $\mathrm{AU}$ & $\mathrm{P}$ & 3 & 27,78 & 2,03 & 41,67 & 3,88 \\
\hline Solanum americanum Mill. & $\mathrm{N}$ & $\mathrm{H}$ & $\mathrm{ZO}$ & $\mathrm{R}$ & 3 & 27,78 & 2,03 & 8,33 & 0,78 \\
\hline Ceiba speciosa (A. St.-Hil.) Ravenna & $\mathrm{N}$ & $\mathrm{L}$ & $\mathrm{AN}$ & $\mathrm{P}$ & 2 & 18,52 & 1,35 & 8,33 & 0,78 \\
\hline Jacaranda micrantha Cham. & $\mathrm{N}$ & $\mathrm{L}$ & AN & $\mathrm{P}$ & 2 & 18,52 & 1,35 & 16,67 & 1,55 \\
\hline Machaerium stipitatum (DC.) Vog. & $\mathrm{N}$ & $\mathrm{L}$ & $\mathrm{AN}$ & $\mathrm{P}$ & 2 & 18,52 & 1,35 & 33,33 & 3,10 \\
\hline Albizia polycephala (Benth.) Killip & $\mathrm{N}$ & $\mathrm{L}$ & $\mathrm{AU}$ & $\mathrm{P}$ & 2 & 18,52 & 1,35 & 8,33 & 0,78 \\
\hline Calliandra tweediei Benth. & $\mathrm{N}$ & $\mathrm{L}$ & $\mathrm{AU}$ & $\mathrm{P}$ & 2 & 18,52 & 1,35 & 8,33 & 0,78 \\
\hline Sebastiania schottiana (Müll. Arg.) Müll. Arg. & $\mathrm{N}$ & $\mathrm{L}$ & $\mathrm{AU}$ & $\mathrm{P}$ & 2 & 18,52 & 1,35 & 33,33 & 3,10 \\
\hline Aegiphyla sp. & $\mathrm{N}$ & $\mathrm{L}$ & $\mathrm{ZO}$ & $\mathrm{P}$ & 2 & 18,52 & 1,35 & 33,33 & 3,10 \\
\hline Campomanesia xanthocarpa O. Berg. & $\mathrm{N}$ & $\mathrm{L}$ & $\mathrm{ZO}$ & $\mathrm{P}$ & 2 & 18,52 & 1,35 & 16,67 & 1,55 \\
\hline Celtis sp. & $\mathrm{N}$ & $\mathrm{L}$ & $\mathrm{ZO}$ & $\mathrm{P}$ & 2 & 18,52 & 1,35 & 16,67 & 1,55 \\
\hline Eugenia pyriformis Cambess. & $\mathrm{N}$ & $\mathrm{L}$ & $\mathrm{ZO}$ & $\mathrm{P}$ & 2 & 18,52 & 1,35 & 8,33 & 0,78 \\
\hline Inga cf. uruguensis Hook. \& Arn. & $\mathrm{N}$ & $\mathrm{L}$ & $\mathrm{ZO}$ & $\mathrm{P}$ & 2 & 18,52 & 1,35 & 33,33 & 3,10 \\
\hline Myrceugenia euosma (O.Berg) D.Legrand. & $\mathrm{N}$ & $\mathrm{L}$ & $\mathrm{ZO}$ & $\mathrm{P}$ & 2 & 18,52 & 1,35 & 16,67 & 1,55 \\
\hline Podocarpus lambertii Klotzsch ex Endl. & $\mathrm{N}$ & $\mathrm{L}$ & $\mathrm{ZO}$ & $\mathrm{P}$ & 2 & 18,52 & 1,35 & 25,00 & 2,33 \\
\hline Rapanea ferruginea (Ruiz \& Pav.) Mez & $\mathrm{N}$ & $\mathrm{L}$ & $\mathrm{ZO}$ & $\mathrm{P}$ & 2 & 18,52 & 1,35 & 25,00 & 2,33 \\
\hline Sloanea monosperma Vell. & $\mathrm{N}$ & $\mathrm{L}$ & $\mathrm{ZO}$ & $\mathrm{P}$ & 2 & 18,52 & 1,35 & 16,67 & 1,55 \\
\hline Strychnos brasiliensis (Spreng.) Mart. & $\mathrm{N}$ & $\mathrm{L}$ & $\mathrm{ZO}$ & $\mathrm{P}$ & 2 & 18,52 & 1,35 & 8,33 & 0,78 \\
\hline Trema micranta (L.) Blume & $\mathrm{N}$ & $\mathrm{L}$ & $\mathrm{ZO}$ & $\mathrm{P}$ & 2 & 18,52 & 1,35 & 66,67 & 6,20 \\
\hline Conyza bonariensis (L.) Cronquist & $\mathrm{N}$ & $\mathrm{H}$ & $\mathrm{AN}$ & $\mathrm{R}$ & 1 & 9,26 & 0,68 & 16,67 & 1,55 \\
\hline Aspidosperma polyneuron Müll. Arg. & $\mathrm{N}$ & $\mathrm{L}$ & AN & $\mathrm{P}$ & 1 & 9,26 & 0,68 & 8,33 & 0,78 \\
\hline Erythrina falcata Benth. & $\mathrm{N}$ & $\mathrm{L}$ & $\mathrm{AN}$ & $\mathrm{P}$ & 1 & 9,26 & 0,68 & 16,67 & 1,55 \\
\hline Ruprechtia laxiflora Meisn. & $\mathrm{N}$ & $\mathrm{L}$ & AN & $\mathrm{P}$ & 1 & 9,26 & 0,68 & 8,33 & 0,78 \\
\hline
\end{tabular}


TABELA 3: Continuação...

TABLE 3: Continuação...

\begin{tabular}{|c|c|c|c|c|c|c|c|c|c|}
\hline Espécies & OR & $\mathrm{FV}$ & SDP & $\mathrm{P} / \mathrm{R}$ & $\mathrm{Ni}$ & Dai & Dri & Fai & Fri \\
\hline Leonurus japonicus Houtt. & $\mathrm{E}$ & $\mathrm{H}$ & $\mathrm{AU}$ & $\mathrm{R}$ & 1 & 9,26 & 0,68 & 8,33 & 0,78 \\
\hline Euphorbia heterophylla $\mathrm{L}$. & $\mathrm{N}$ & $\mathrm{H}$ & $\mathrm{AU}$ & $\mathrm{R}$ & 1 & 9,26 & 0,68 & 66,67 & 6,20 \\
\hline Peltophorum dubium (Spreng.) Taub. & $\mathrm{N}$ & $\mathrm{L}$ & $\mathrm{AU}$ & $\mathrm{P}$ & 1 & 9,26 & 0,68 & 8,33 & 0,78 \\
\hline $\begin{array}{l}\text { Sebastiania commersoniana (Baill.) } \\
\text { L.B.Sm. \& Downs. }\end{array}$ & $\mathrm{N}$ & $\mathrm{L}$ & $\mathrm{AU}$ & $\mathrm{P}$ & 1 & 9,26 & 0,68 & 16,67 & 1,55 \\
\hline $\begin{array}{l}\text { Tabebuia chrysotricha (Mart. Ex A.DC.) } \\
\text { Standl. }\end{array}$ & $\mathrm{N}$ & $\mathrm{L}$ & $\mathrm{AU}$ & $\mathrm{P}$ & 1 & 9,26 & 0,68 & 25,00 & 2,33 \\
\hline Alchornea sp. & $\mathrm{N}$ & $\mathrm{L}$ & $\mathrm{ZO}$ & $\mathrm{P}$ & 1 & 9,26 & 0,68 & 25,00 & 2,33 \\
\hline Annona cacans Warm. & $\mathrm{N}$ & $\mathrm{L}$ & $\mathrm{ZO}$ & $\mathrm{P}$ & 1 & 9,26 & 0,68 & 16,67 & 1,55 \\
\hline Casearia decandra Jacq. & $\mathrm{N}$ & $\mathrm{L}$ & $\mathrm{ZO}$ & $\mathrm{P}$ & 1 & 9,26 & 0,68 & 16,67 & 1,55 \\
\hline Eugenia involucrata DC. & $\mathrm{N}$ & $\mathrm{L}$ & $\mathrm{ZO}$ & $\mathrm{P}$ & 1 & 9,26 & 0,68 & 16,67 & 1,55 \\
\hline Ficus enormis (Mart. Ex Miq.) Mart & $\mathrm{N}$ & $\mathrm{L}$ & $\mathrm{ZO}$ & $\mathrm{P}$ & 1 & 9,26 & 0,68 & 8,33 & 0,78 \\
\hline Psidium cf. cattleyanum Sabine & $\mathrm{N}$ & $\mathrm{L}$ & $\mathrm{ZO}$ & $\mathrm{P}$ & 1 & 9,26 & 0,68 & 16,67 & 1,55 \\
\hline Rapanea umbellata (Mart.) Mez & $\mathrm{N}$ & $\mathrm{L}$ & $\mathrm{ZO}$ & $\mathrm{P}$ & 1 & 9,26 & 0,68 & 8,33 & 0,78 \\
\hline Xylosma sp. & $\mathrm{N}$ & $\mathrm{L}$ & $\mathrm{ZO}$ & $\mathrm{P}$ & 1 & 9,26 & 0,68 & 8,33 & 0,78 \\
\hline Zanthoxylum rhoifolium Lam. & $\mathrm{N}$ & $\mathrm{L}$ & $\mathrm{ZO}$ & $\mathrm{P}$ & 1 & 9,26 & 0,68 & 16,67 & 1,55 \\
\hline Total & & & & & 148 & 1370,37 & 100,00 & 1075,00 & 100,00 \\
\hline
\end{tabular}

Em que: $\mathrm{OR}=$ origem $(\mathrm{N}=$ nativa, $\mathrm{E}=$ exótica, $\mathrm{IN}=$ indeterminada $), \mathrm{FV}=$ forma de vida $(\mathrm{H}=$ herbácea, $\mathrm{L}=$ lenhosa, IN = indeterminada), $\mathrm{SDP}=$ síndrome de dispersão $(\mathrm{AN}=$ anemocoria, $\mathrm{AU}=$ autocoria, $\mathrm{ZO}=$ zoocoria, $\mathrm{IN}$ $=$ indeterminada $), \mathrm{P} / \mathrm{R}=$ plantada ou regenerante $(\mathrm{P}=$ plantada, $\mathrm{R}=$ regenerante $), \mathrm{Ni}=$ número de indivíduos, $\mathrm{Dai}=$ densidade absoluta $\left(\right.$ Nind.ha $\left.^{-1}\right)$, Dri $=$ densidade relativa $(\%), F a i=$ frequência absoluta $(\%)$, Fri $=$ frequência relativa $(\%)$.

\section{Comparação entre os tratamentos: indivíduos plantados e regenerantes}

A julgar os indivíduos regenerantes e plantados houve diferença significativa entre o número médio de indivíduos dos tratamentos PA $(504,5)$ e PL (37) (Tabela 4). Trata-se de uma diferença relativamente grande, o que reflete nos tratos culturais aplicados no PL durante os três primeiros anos. Não houve diferença significativa entre o número de indivíduos da nucleação e do plantio, devido à alta variação das parcelas de nucleação.

TABELA 4: Quadro de médias e desvio padrão considerando plantados e regenerantes. Médias seguidas de letras iguais não diferem pelo teste de Tukey a $5 \%$ de probabilidade.

TABLE 4: Table of means and standard deviation considering planted and regenerated. Means followed by the same letter do not differ by Tukey test at $5 \%$ probability.

\begin{tabular}{ccccc}
\hline & $\mathrm{Ni}$ & $\mathrm{S}$ & $\mathrm{H}$ & $\mathrm{J}$ \\
\hline $\mathrm{NU}$ & $336,25 \pm 204,94 \mathrm{ab}$ & $25,5 \pm 11,62 \mathrm{a}$ & $2,008 \pm 0,949 \mathrm{a}$ & $0,612 \pm 0,218 \mathrm{~b}$ \\
$\mathrm{PA}$ & $504,5 \pm 243,78 \mathrm{a}$ & $19,0 \pm 4,69 \mathrm{a}$ & $1,965 \pm 0,098 \mathrm{a}$ & $0,674 \pm 0,024 \mathrm{ab}$ \\
$\mathrm{PL}$ & $37,0 \pm 9,93 \mathrm{~b}$ & $24,0 \pm 4,97 \mathrm{a}$ & $3,017 \pm 0,156 \mathrm{a}$ & $0,956 \pm 0,038 \mathrm{a}$
\end{tabular}

Em que: $\mathrm{Ni}$ = média do número de indivíduos; $\mathrm{S}$ = média da riqueza de espécies; $\mathrm{H}^{\prime}=$ índice de Shannon ( $\mathrm{H}^{\prime}-$ nats. indv- $\left.^{1}\right) ; \mathrm{J}=$ equabilidade de Pielou.

A riqueza média de espécies não diferiu significativamente, assim como para o índice de diversidade de Shannon entre os tratamentos, mas o PL apresentou o maior valor para este índice $(3,017)$ um valor similar ao encontrado em estudos de florestas maduras (SOUZA et al., 2012; FIORENTIN et al., 2015) o que ocorre provavelmente devido ao grande número de espécies implantadas neste tratamento. A alta equabilidade do tratamento PL foi resultado do baixo número de indivíduos proporcionalmente ao número 
de espécies, de forma que uma área com $\mathrm{J}=1$ significaria que todas as espécies daquela comunidade são igualmente abundantes.

Analisando-se o índice de similaridade de Jaccard, pode-se observar que os tratamentos PA e NU possuem a maior similaridade $(0,28)$ entre os tratamentos (Tabela 5). A menor similaridade pode ser observada entre a PA e o PL $(0,09)$.

TABELA 5: Índice de similaridade de Jaccard obtidos através da comparação dos indivíduos plantados e regenerantes dos três tratamentos no município de Dois Vizinhos - PR.

TABLE 5: Jaccard similarity index obtained through comparison of planted and regenerated individuals of the three treatments in Dois Vizinhos - PR state.

\begin{tabular}{lc}
\hline \multicolumn{1}{c}{ Comparação } & Índice de similaridade de Jaccard \\
\hline Restauração passiva x Nucleação & 0,28 \\
Nucleação x Plantio em linhas de recobrimento e diversidade & 0,17 \\
Restauração passiva x Plantio em linhas de recobrimento e diversidade & 0,09 \\
\hline
\end{tabular}

Esta menor similaridade observada entre os tratamentos PA e PL, ocorre devido ao fato de que no tratamento PA, o nível de interferência é praticamente nulo, porque não houve a introdução de espécies, já no PL a introdução de mudas presselecionadas ocorreu em área total combinada com manutenções, não restando espaço para a regeneração de indivíduos que poderiam assemelhar-se a uma sucessão secundária natural, a qual ocorre na PA. A NU apresenta comportamento intermediário devido ao espaço entre técnicas que permite a ocorrência de uma maior regeneração de espécies.

\section{Comparação entre os tratamentos: indivíduos regenerantes}

Haja vista apenas os indivíduos regenerantes de cada tratamento, novamente houve diferença significativa apenas entre o número médio de indivíduos da PA e do PL (504,5 e 8,75, respectivamente) (Tabela 6). Não houve diferença significativa entre o número de indivíduos médio da NU com os demais tratamentos.

TABELA 6: Quadro de médias e desvio padrão considerando somente regenerantes. Médias seguidas de letras iguais não diferem pelo teste de Tukey a 5\% de probabilidade.

TABLE 6: Table of means and standard deviation considering only regenerated. Means followed by the same letter do not differ by Tukey test at $5 \%$ probability.

\begin{tabular}{ccccc}
\hline & $\mathrm{Ni}$ & $\mathrm{S}$ & $\mathrm{H}$ & $\mathrm{J}$ \\
\hline $\mathrm{NU}$ & $227 \pm 182,11 \mathrm{ab}$ & $18,5 \pm 10,72 \mathrm{a}$ & $2,078 \pm 0,657 \mathrm{a}$ & $0,794 \pm 0,148 \mathrm{ab}$ \\
$\mathrm{PA}$ & $504,5 \pm 243,78 \mathrm{a}$ & $19,0 \pm 4,69 \mathrm{a}$ & $1,965 \pm 0,098 \mathrm{ab}$ & $0,674 \pm 0,024 \mathrm{~b}$ \\
$\mathrm{PL}$ & $8,75 \pm 8,92 \mathrm{~b}$ & $4,0 \pm 1,63 \mathrm{~b}$ & $1,166 \pm 0,360 \mathrm{~b}$ & $0,899 \pm 0,090 \mathrm{a}$ \\
\hline
\end{tabular}

Em que: $\mathrm{Ni}$ = média do número de indivíduos; $\mathrm{S}=$ média da riqueza de espécies; $\mathrm{H}^{\prime}$ = índice de Shannon $\left(\mathrm{H}^{\prime}-\right.$ nats. indv- $\left.^{-1}\right) ; \mathrm{J}=$ equabilidade de Pielou.

Quanto à riqueza média de espécies, o tratamento PL foi significativamente diferente dos demais por apresentar o menor número de espécies (4). O PL também apresentou o menor índice de diversidade de Shannon $(1,166)$ e foi significativamente diferente da NU $(2,078)$ a qual apresentou a maior diversidade. A PA não diferiu de nenhum tratamento em relação ao índice de diversidade de Shannon. O fato do tratamento PL possuir uma menor diversidade em relação aos indivíduos regenerantes ocorre em decorrência dos tratos culturais aplicados como parte do tratamento, incluindo roçagem da área e aplicação de herbicida, sendo estas manutenções realizadas até os três anos de idade (espera-se que em um futuro próximo ocorra a regeneração do sub-bosque formado). Estas mesmas manutenções foram aplicadas no tratamento NU, porém somente nas faixas onde foram implantadas as técnicas ( $1 / 3$ da área), permitindo assim a ocorrência da regeneração natural no restante da área. O PL novamente apresentou uma equabilidade próxima de um, e a nucleação apresentou um aumento na equabilidade quando considerados apenas indivíduos regenerantes, 
o que indica que alguma espécie implantada provavelmente apresentou certa dominância em número de indivíduos.

A similaridade (Tabela 7) foi maior entre os tratamentos PA e NU $(0,3559)$ e menor entre os tratamentos NU e PL $(0,1395)$.

TABELA 7: Índice de similaridade de Jaccard obtidos através da comparação dos indivíduos regenerantes dos três tratamentos no município de Dois Vizinhos - PR.

TABLE 7: Jaccard similarity index obtained through comparison of regenerated individuals of the three treatments in Dois Vizinhos - PR.

\begin{tabular}{lc}
\hline \multicolumn{1}{c}{ Comparação } & Índice de similaridade de Jaccard \\
\hline Restauração passiva x Nucleação & 0,36 \\
Nucleação x Plantio em linhas de recobrimento e diversidade & 0,14 \\
Restauração passiva x Plantio em linhas de recobrimento e diversidade & 0,20 \\
\hline
\end{tabular}

O PL foi o menos similar tanto com a NU como com a PA, e isto ocorreu provavelmente porque a densidade de indivíduos regenerantes no plantio foi muito baixa, devido ao grande número de árvores plantadas, roçagens e capinas químicas realizadas como parte do tratamento. Desta forma, não houve habitat favorável para indivíduos regenerantes, sendo que estes ocorreram em baixas densidades.

\section{CONCLUSÃO}

Analisando-se os indivíduos plantados e regenerantes de todos os tratamentos, o plantio em linhas (PL) apresentou a maior diversidade de espécies, porém, não houve diferença significativa. Ao avaliar apenas os indivíduos regenerantes, a nucleação (NU) apresentou a maior diversidade seguida da restauração passiva (PA) e por último o plantio (PL) o qual diferiu significativamente da NU. Apesar do fato de que o PL proporcionou uma maior diversidade em estágio inicial de restauração (considerando plantados e regenerantes) se comparado aos demais tratamentos, a utilização desta técnica mostrou-se pouco eficiente quanto à presença de regeneração natural, devido ao fato da necessidade de manutenção destas áreas para garantir a sobrevivência das mudas plantadas e também ao grande número de mudas introduzidas, afetando os rumos naturais de sucessão. Este tratamento também demanda um orçamento maior, devido ao grande número de mudas implantadas (1.666 mudas.ha $\left.{ }^{-1}\right)$ e também dos procedimentos silviculturais necessários para manutenção da área até os três anos de idade. A técnica de nucleação, que exibiu uma diversidade semelhante à PA, apresenta custos menores devido ao fato de que as técnicas são implantadas em apenas $1 / 3$ da área. Já a PA não apresenta custos experimentais de implantação (em uma condição real, este tratamento, assim como os demais, muitas vezes exige a construção de cercas e patrulhamento). No local em que este estudo foi realizado, a utilização da restauração passiva pode ser considerada uma boa opção, dado que seus resultados não se diferiram estatisticamente da nucleação, e a principal vantagem desta técnica é o custo reduzido.

\section{AGRADECIMENTOS}

À Fundação Araucária (bolsa PIBIC-af), ao Conselho Nacional de Desenvolvimento Científico e Tecnológico - CNPq (Processo 575081/2008-2) pelo apoio financeiro, e à COPEL-GET (especialmente ao Dr. Murilo Barddal), IAP e De Bortoli Madeiras pelo apoio logístico.

\section{REFERÊNCIAS}

AIDE, T. M. et al. Forest regeneration in a chronosequence of tropical abandoned pastures: implications for restoration ecology. Restoration Ecology, Washington, v. 8, n. 4, p. 328-338, dez. 2000.

BECHARA, F. C. et al. Neotropical rainforest restoration: comparing passive, plantation and nucleation approaches. Biodiversity and Conservation, Amsterdam, v. 25, n. 11, p. 2021-2034, jul. 2016. 
BOANARES, D.; AZEVEDO, C. S. The use of nucleation techniques to restore the environment: a bibliometric analysis. Natureza e Conservação, Ouro Preto, v. 12, n. 2, p. 93-98, 2014.

BRANCALION, P. S.; RODRIGUES, R. R.; GANDOLFI, S. Restauração florestal. 1. ed. São Paulo: Oficina de Textos, 2015. $432 \mathrm{p}$.

BRASIL. Ministério da Agricultura, Pecuária e Abastecimento. Plano setorial de mitigação e de adaptação às mudanças climáticas para a consolidação de uma economia de baixa emissão de carbono na agricultura: plano ABC (Agricultura de Baixa Emissão de Carbono). Brasília: MAPA, 2012. 173 p.

CORBIN, J. D.; HOLL, K. D. Applied nucleation as a forest restoration strategy. Forest Ecology and Management, Netherlands, v. 265, p. 37-46, 2012.

ENGEL, V. L.; PARROTTA, J. A. Definindo a restauração ecológica: tendências e perspectivas mundiais. In: KAGEYAMA, P. Y. et al. (Org.). Restauração ecológica de ecossistemas naturais. Botucatu: Fundação de Estudos e Pesquisas Agrícolas e Florestais, 2003. p. 3-26.

FIORENTIN, L. D. et al. Análise florística e padrão espacial da regeneração natural em área de Floresta Ombrófila Mista na região de Caçador, SC. Floram, Seropédica, v. 22, n. 1, p. 60-70, 2015.

GOMEZ-APARICIO, L. et al. Applying plant facilitation to forest restoration: a meta-analysis of the use of shrubs as nurse plants. Ecological Applications, Tempe, v. 14, n. 4, p. 1128-1138, 2004.

HOLL, K. D.; AIDE, T. M. When and where to actively restore ecosystems? Forest Ecology and Management, Netherlands, v. 261, p. 1558-1563, 2011.

HOWE, H. F.; SMALLWOOD, J. Ecology of seed dispersal. Annual Review of Ecology, Evolution and Systematics, Palo Alto, v. 13, p. 201-228, 1982.

INSTITUTO NACIONAL DE PESQUISAS ESPACIAIS (Brasil). SOS Mata Atlântica e INPE apresentam dados do Atlas dos Remanescentes Florestais da Mata Atlântica. 2014. Disponível em: $<$ http://www.inpe.br/noticias/noticia.php?Cod_Noticia=3610>. Acesso em: 7 nov. 2015.

LIEBSCH, D.; ACRA, L. A. Riqueza de espécies de sub-bosque de um fragmento de Floresta Ombrófila Mista em Tijucas do Sul - PR. Ciência Florestal, Santa Maria, v. 14, n. 1, p. 67-76, 2004.

MORRISON, E. B.; LINDELL, C. A. Active or passive forest restoration? assessing restoration alternatives with avian foraging behavior. Restoration Ecology, Washington, v. 19, n. 201, p. 170-177, mar. 2011.

PIJL, L. van der. Principles of dispersal in higher plants. 3rd ed. New York: Springer-Verlag, 1982. 402 p. REIS, A.; BECHARA, F. C.; TRES, D. R. Nucleation in tropical ecological restoration. Scientia Agricola, Piracicaba, v. 67, n. 2, p. 244-250, mar./abr. 2010.

REIS, A. et al. Nucleação: concepção biocêntrica para a restauração ecológica. Ciência Florestal, Santa Maria, v. 24, n. 2, p. 509-518, abr./jun. 2014.

RODRIGUES, R. R. et al. Large-scale ecological restoration of high-diversity tropical forests in SE Brazil. Forest Ecology and Management, Netherlands, v. 261, p. 1605-1613, 2011.

RODRIGUES, R. R. et al. On the restoration of high diversity forests: 30 years of experience in the Brazilian Atlantic Forest. Biological Conservation, Boston, v. 142, n. 6, p. 1242-1251, 2009.

SOUZA, R. P. M. et al. Estrutura e aspectos da regeneração natural de Floresta Ombrófila Mista no Parque Estadual de Campos do Jordão, SP, Brasil. Hoehnea, São Paulo, v. 39, n. 3, p. 387-407, 2012.

VOGEL, H. F.; CAMPOS, J. B.; BECHARA, F. C. Early bird assemblages under different subtropical forest restoration strategies in Brazil: passive, nucleation and high diversity plantation. Tropical Conservation Science, [s. 1.], v. 8, n. 4, p. 912-939, 2015.

WADT, P. G. S. Práticas de conservação do solo e recuperação de áreas degradadas. Rio Branco: Embrapa Acre, 2003. 